\title{
Immunohistochemical expression of tenascin and elastin in women with pelvic organ prolapse and/or without stress urinary incontinence
}

\author{
Ilias Liapis ${ }^{1}$, Panagiotis Bakas ${ }^{2}$, Pafiti-Kondi Agatha ${ }^{3}$, Matrona Frangou-Plemenou ${ }^{4}$, Charalampos Karachalios ${ }^{5 *}$, Dimos Sioutis ${ }^{6}$ and $^{2}$ \\ Aggelos Liapis ${ }^{2}$ \\ ${ }^{1}$ Birmingham Women's and Children's Hospital, NHS Foundation Trust, Health Education England Midlands and East-West Midlands, Birmingham, England, \\ United Kingdom \\ ${ }^{2}$ Second Department of Obstetrics and Gynecology, Aretaieio University Hospital, School of Health Sciences, Medical School, National and Kapodistrian University \\ of Athens, Athens, Attica, Greece \\ ${ }^{3}$ Pathology Laboratory, Aretaieio University Hospital, School of Health Sciences, Medical School, National and Kapodistrian University of Athens, Athens, Attica, \\ Greece \\ ${ }^{4}$ Microbiology Laboratory, Aretaieio University Hospital, School of Health Sciences, Medical School, National and Kapodistrian University of Athens, Athens, \\ Attica, Greece \\ ${ }^{5}$ Second Department of Obstetrics and Gynecology, Aretaieio University Hospital, School of Health Sciences, Medical School, National and Kapodistrian University \\ of Athens, Athens, Attica, Greece \\ ${ }^{6}$ Third Department of Obstetrics and Gynecology, Attikon University Hospital, School of Health Sciences, Medical School, National and Kapodistrian University \\ of Athens, Athens, Attica, Greece
}

\begin{abstract}
Background and aim: Pelvic organ prolapse (POP) and stress urinary incontinence (SUI) constitute entities of pelvic floor disorders and most often occur simultaneously in the same patient, adversely affecting women's quality of life. The pathogenesis of pelvic organ prolapse and stress urinary incontinence is not fully understood. The pelvic viscera are maintained in their place thanks to interconnection of levator ani muscles, cardinal and uterosacral ligaments, and pubocervical and rectovaginal fascia. Ligaments and fascia consist mainly of connective tissue. Alterations in the metabolism of its extracellular matrix could contribute POP and SUI. The aim of this study was to assess the metabolism of extracellular matrix components in relation to the immunohistochemical expression of tenascin and elastin in patients with POP and/or without SUI.

Methods: In the present study, we included 90 adult Greek women; 30 women with POP and SUI, 30 women with POP and no SUI, and 30 controls. For the immunohistochemical study, 90 biopsies were obtained after hysterectomy from the vagina (pubocervical fascia) and the attachment areas of uterosacral and cardinal ligaments. The specimens were studied for immunohistochemical evaluation of tenascin and elastin.

Results: Tenascin was strongly expressed in all samples obtained from women with prolapse. Patients with total prolapse showed enhanced immunoactivity to the tenascin-specific antigen near connective tissue and around the wall of vessels. Continent patients with only POP and the control group were more likely to have weakly positive or negative tenascin immunoreaction in stromal cells and local expression in muscles/vessels. Regarding the immunohistochemical response of elastin expression, we observed an increased number of patients with reduced elastin and decreased number of patients with positive expression of elastin in patients with prolapse and SUI.
\end{abstract}

Conclusions: These alterations on the metabolism of extracellular matrix components of the connective tissue seem to contribute to the pathogenesis of POP and SUI and may aid in non-surgical treatment of these disorders.

\section{Introduction}

The term "pelvic floor disorders" includes a wide range of clinical manifestations. Pelvic floor relaxation and urinary incontinence are parts of the above general term. Pelvic floor disorders degrade women's quality of life and pose an economic burden to individuals. They greatly affect both developing and developed countries [1].

Pelvic floor disorders are quite common. It has been estimated that one third of premenopausal women have a pelvic floor disorder and about half of postmenopausal. Most therapeutical interventions cannot drastically solve the problem of both prolapse and incontinence. Recurrence rate for relaxation and stress urinary incontinence (SUI) have been estimated at about $29 \%$ and $15 \%$ respectively, for a five-year follow-up [2]. The pathogenesis of pelvic organ prolapse (POP) and SUI is not fully known. It is multidisciplinary and multilevel.

${ }^{*}$ Correspondence to: Charalampos Karachalios, Miltiadou 14, 14231, Nea Ionia, Athens, Greece, E-mail: harrykarachalios@windowslive.com

Key words: collagen metabolism, pelvic organ prolapse, stress urinary incontinence, tenascin, elastin

Received: April 03, 2021; Accepted: April 16, 2021; Published: April 24, 2021 
The pelvic organs maintain their position thanks to their supportive connection to the elevator ani muscles, the cardinal and uterosacral ligaments, and the pubocervical and rectovaginal fascia. Loss of elevator ani muscles' function is a key factor for pelvic organ prolapse [3].

The ligaments and fascia mainly consist of connective tissue. Alterations of their metabolism could be responsible for the development of POP and SUI. Pelvic floor trauma is encountered in a large proportion of patients with pelvic organ prolapse and stress urinary incontinence. However, a group of this patients does not have any anatomical damage. Furthermore, cesarean section does not completely diminish the risk of relaxation. Genetic factors could play a significant role in the development of pelvic organ prolapse.

The ligamentous support of the pelvic floor is affected by the functions of the extracellular matrix in the connective tissue. Therefore, part of current research concerning POP and SUI has been focused on the metabolism of the extracellular matrix.

Extracellular matrix consists of different combinations of longchain protein fibers (including predominantly collagen, reticular and elastic tissue) and ground substance, which is composed of glycozaminoglycans, proteoglycans and multi-adhesive glycoproteins, such as tenascin, relaxin, fibulin and elastin [4]. Extracellular matrix is maintained from fibroblasts through secretion of proteases and growth factors, which modify the synthesis and breakdown of structural fibers [5]. The objective of the present study is to assess the changes in the quantity in the immunolocalization of elastin and tenascin in patients with genital prolapse and stress urinary incontinence with controls.

\section{Material and methods}

All participant females in the present study were submitted to vaginal hysterectomy for uterine prolapse (stages I-IV). Each woman was categorized into one of three groups. Group A consisted of 30 patients with stress urinary incontinence and prolapse. Group B had 30 patients with prolapse, but without stress urinary incontinence. Group C consisted of 30 women without uterine prolapse and no SUI and these patients were submitted to abdominal hysterectomy for benign conditions (e.g. leiomyomas). Classification of pelvic floor prolapse was performed with the use of pelvic organ prolapse quantification system (POP-Q system). Stage 0 is the gold standard for normal vaginal support. Patients with a history of malignancy of the urogenital system, connective tissue abnormalities, emphysema, previous history of pelvic surgery and estrogen administration within 6 months of the procedure were excluded from the study. The initial gynecological examination was done by the Head of the Urogynecology Department of the hospital. Patients' written consent was obtained and the study was approved by the Research and Ethics committee of "Aretaieion" University Hospital in Athens. All patients were examined in the lithotomy position with a full bladder and were asked to perform the Valsalva maneuver and cough repeatedly for 3 to 5 times, to assess for the presence of stress incontinence. Moreover, after emptying the bladder and performing the Valsalva maneuver, patients were assessed for degree of prolapse. The descend of the vaginal parts was measured at maximum intensity using a metric rule. The total length of the vagina was measured at rest and reduction of relaxation using anterior and posterior single-bladed vaginal specula. The patients were then examined in erect position to confirm the full degree of prolapse.

For the immunopathological study, 90 biopsies, measuring $5 \times 5$ $\mathrm{mm}$, were obtained after hysterectomy with Metzenbaum scissors from the vagina (pubocervical fascia) and from the attachment sites of uterosacral and cardinal ligaments of the uterus.
The specimens were fixed in $5 \%$ formalin solution and were embedded in paraffin, following the standard process for histopathology. Hematoxylin-eosin-stained sections were studied and additional sections were obtained for immunohistological evaluation of tenascin and elastin expression.

A semi-automatic immunohistochemistry Ventana ${ }^{\text {Txx }}$ system was used in accordance to manufacturer guidance with elastin and tenascin antibodies [anti-elastin antibody (ab21610), rabbit polyclonal to elastin, $\mathrm{Abcam}^{\mathrm{Tw}}$, Cambridge, UK, and Tenascin-C monoclonal antibodies BC27, Introgen Therapeutics ${ }^{\text {Txx }}$ Inc, USA).

The evaluation of immunostaining was performed by two observers using a semi-quantitative system and was recorded as negative (0) when less than $10 \%$ of the cells showed positive immunoreactions, as positive (1) when more than $10 \%$ of the cells were stained and strongly positive (2) when more than $30 \%$ of the cells were stained. The distribution of antibodies in the cytoplasm, the membrane and the nucleus of cells were recorded separately. Furthermore, the location of immunostained cells in the stroma, round vessels, and in muscle bundles or subepithelial areas was recorded.

\section{Statistical analysis}

The statistical analysis of the 90 individuals in the sample includes descriptive and inferential statistics of their measurements. In terms of descriptive statistics, absolute and relative (\%) frequencies were used for quality variables. Bar graphs were used to graphically represent the data. In the inferential statistics, the exact control $\mathrm{X}^{2}$ test was used for the investigation of relations between qualitative variables. Results with P-value less than 0.05 are considered statistically significant. The statistical analysis was performed with the $25^{\text {th }}$ version of SPSS ${ }^{\text {six }}($ IBM).

\section{Results}

There were no statistically significant differences between the groups of patients (Groups A and B) and the group of controls (Group $\mathrm{C})$, regarding to age, Body Mass Index (BMI) and smoking $(\mathrm{P}>0.05)$, as shown in Table 1.

We used the $\mathrm{X}^{2}$ test and Fisher's exact test to check if there is a relationship between the groups and the expression of tenascin. The calculated P-value for both tests was less than 0.001 , which is statistically significant (Table 2).

The structure of tenascin was labeled in all samples. Weak expression of tenascin was observed in samples taken from women without pelvic relaxation. Tenascin immunohistochemistry expression per Group is summarized in Graph 1.

Tenascin was strongly labeled in all samples obtained from women with relaxation. Patients with IV degree prolapse showed enhanced immunoactivity to the tenascin-specific antigen, near the connective tissue and around the wall of vessels. Therefore, there was a statistically significant relationship between group and tenascin, specifically in patients with prolapse and incontinence (Figure 1).

(weakly positive) tenascin immunoreactions in stromal cells and local expression in muscles / vessels in patients without prolapse and stress urinary incontinence was observed (Figure 2, Group C).

In continent patients with prolapse (Group B), the observed values of tenascin are close to expected, i.e. to the patients with POP and SUI. The relationship between groups and elastin was studied using the control $\mathrm{X}^{2}$ test. The calculated P-value was less than 0.001 , which is statistically significant. Thus, there is a statistically significant 
Table 1. Demographic characteristics of patients and controls

\begin{tabular}{|c|c|c|c|c|c|c|c|c|c|c|}
\hline & & \multicolumn{3}{|c|}{ Group } & \multicolumn{6}{|c|}{ P-value } \\
\hline & & $\mathbf{A}$ & $\mathbf{B}$ & $\mathbf{C}$ & Total & All groups & $A-(B+C)$ & A-C & A-B & B-C \\
\hline \multicolumn{11}{|c|}{ Patients Characteristics } \\
\hline & $46-60$ & $12(40 \%)$ & $13(43.3 \%)$ & $13(42.2 \%)$ & $38(42.2 \%)$ & \multirow{3}{*}{1} & \multirow{3}{*}{1} & \multirow{3}{*}{1} & \multirow{3}{*}{1} & \multirow{3}{*}{1} \\
\hline \multirow[t]{3}{*}{ Age } & $61-70$ & $10(33.3 \%)$ & $10(33.3 \%)$ & $9(32.2 \%)$ & $29(32.2 \%)$ & & & & & \\
\hline & $>71$ & $8(26.7 \%)$ & $7(23.3 \%)$ & $8(25.6 \%)$ & $23(25.6 \%)$ & & & & & \\
\hline & Total & $30(100 \%)$ & $30(100 \%)$ & $30(100 \%)$ & $90(100 \%)$ & & & & & \\
\hline & & & & & & & & & & \\
\hline & $18-25$ & $21(70 \%)$ & $22(73.3 \%)$ & $21(70 \%)$ & $64(71.1 \%)$ & \multirow{3}{*}{1} & \multirow{3}{*}{0,911} & \multirow{3}{*}{1} & \multirow{3}{*}{1} & \multirow{3}{*}{1} \\
\hline \multirow[t]{3}{*}{ BMI } & $25-29$ & $8(26.7 \%)$ & $7(23.3 \%)$ & $8(26.7 \%)$ & $23(25.6 \%)$ & & & & & \\
\hline & $>29$ & $1(3.3 \%)$ & $1(3.3 \%)$ & $1(3.3 \%)$ & $3(3.3 \%)$ & & & & & \\
\hline & Total & $30(100 \%)$ & $30(100 \%)$ & $30(100 \%)$ & $90(100 \%)$ & & & & & \\
\hline & & & & & & & & & & \\
\hline & Yes & $12(40 \%)$ & $14(46.7 \%)$ & $13(43.3 \%)$ & $39(43.3 \%)$ & \multirow{2}{*}{0,963} & \multirow{2}{*}{0,822} & \multirow{2}{*}{1} & \multirow{2}{*}{0,795} & \multirow{2}{*}{1} \\
\hline \multirow[t]{2}{*}{ Smoking } & No & $18(60 \%)$ & $16(53.3 \%)$ & $17(56.7 \%)$ & $51(56.7 \%)$ & & & & & \\
\hline & Total & $30(100 \%)$ & $30(100 \%)$ & $30(100 \%)$ & $90(100 \%)$ & & & & & \\
\hline
\end{tabular}

Table 2. Immunohistochemical expression of tenascin in patients and controls

\begin{tabular}{|c|c|c|c|c|c|c|c|c|c|c|}
\hline & \multicolumn{4}{|c|}{ Group } & \multicolumn{6}{|c|}{ П - Value } \\
\hline & \multicolumn{2}{|c|}{$\mathbf{A}$} & B & C & Total & All groups & $A-(B+C)$ & A-C & A-B & B-C \\
\hline \multicolumn{11}{|l|}{ Tenascin } \\
\hline \multirow{4}{*}{$\begin{array}{l}\text { Expression } \\
\text { intensity }\end{array}$} & -- & $7(23,3 \%)$ & $12(40,0 \%)$ & $22(73,3 \%)$ & $41(45,5 \%)$ & & & & & \\
\hline & + & $23(76,7 \%)$ & $18(60,0 \%)$ & $8(26,7 \%)$ & $49(54,5 \%)$ & $<0,001$ & 0,003 & $<0,001$ & 0,267 & 0,018 \\
\hline & ++ & 0 & 0 & 0 & & & & & & \\
\hline & Total & $30(33,3 \%)$ & $30(33,3 \%)$ & $30(33,3 \%)$ & $90(100 \%)$ & & & & & \\
\hline
\end{tabular}

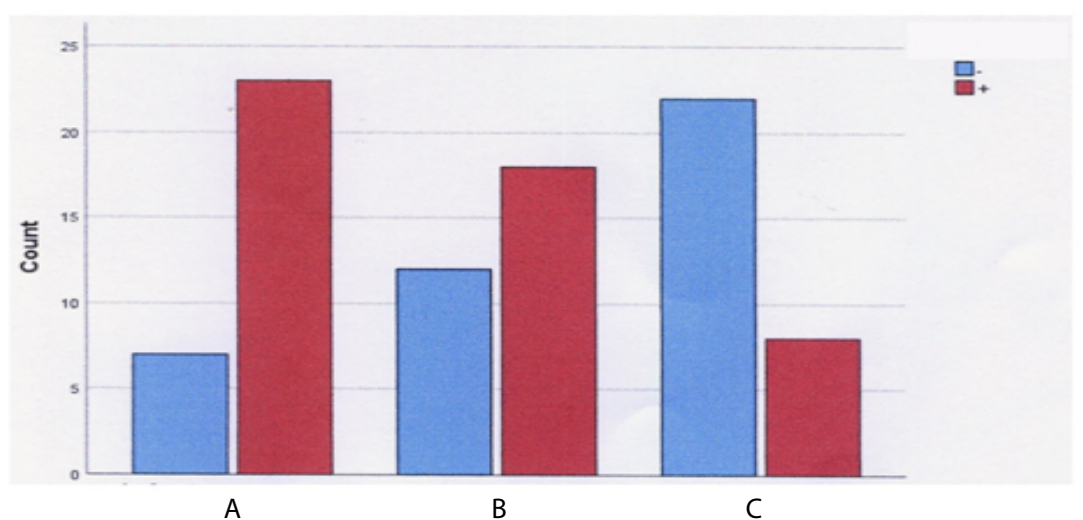

Graph 1. Tenascin expression bar graph per Group (Group A: Patients with POP and SUI, Group B: Patients with POP and no SUI, Group C: controls)

relationship between group and elastin. The labeling pattern of elastin is summarized in Graph 2.

Regarding the immunohistochemical response of elastin expression, there was a significant relationship between groups and elastin, especially in patients with prolapse and stress urinary incontinence. We observed an increased number of patients with reduced elastin and decreased number of patients with positive expression of elastin (Table $3)$.

In patients with POP and SUI (Group A), we observed an increased number of patients with elastin(-) and decreased number of patients with elastin(+) (Figure 3). We also observed an increase to expression of elastin in all patients without SUI and POP (Figure 4). Furthermore, the observed values in continent patients with POP are close to the patients without POP and SUI.

\section{Discussion}

The incidence of pelvic floor dysfunction is high in women of both developing and developed countries [1]. Pelvic floor relaxation is a devastating condition that affects a woman's quality of life. It is argued that pelvic floor support is maintained by interactions between the vaginal wall, the rectum, the levator ani muscles and urethral connective tissue. Loss of elevator ani function is a key factor in pelvic floor prolapse [3].

Previous studies have revealed that mechanical stretching indirectly alters the morphology and arrangement of cells and simulates the expression and secretion of elements of the extracellular matrix, such as collagen type I and type III, tenascin, elastin, lysyl oxidase and Fibulin-5 in bone marrow mesenchymal stem cells cultures [6]. 
Liapis I (2021) Immunohistochemical expression of tenascin and elastin in women with pelvic organ prolapse and/or without stress urinary incontinence

Table 3. Immunohistochemical expression of elastin in patients and controls

\begin{tabular}{|c|c|c|c|c|c|c|c|c|c|c|}
\hline & & \multicolumn{3}{|c|}{ Group } & \multicolumn{6}{|c|}{ P - Value } \\
\hline & & $\mathbf{A}$ & B & $\mathrm{C}$ & Total & All groups & $A-(B+C)$ & A-C & A-B & B-C \\
\hline \multicolumn{11}{|c|}{ Elastin } \\
\hline \multirow{4}{*}{$\begin{array}{c}\text { Expression } \\
\text { intensity }\end{array}$} & - & $26(86,7 \%)$ & $16(53,3 \%)$ & $11(36,7 \%)$ & $53(58,9 \%)$ & & & & & \\
\hline & + & $4(13,6 \%)$ & $14(46,7 \%)$ & $19(63,3 \%)$ & $37(41,1 \%)$ & & $<0,001$ & $<0,001$ & $<0,001$ & 0,299 \\
\hline & ++ & 0 & 0 & 0 & 0 & & & & & \\
\hline & Total & $30(33,3 \%)$ & $30(33,3 \%)$ & $30(33,3 \%)$ & $90(100 \%)$ & & & & & \\
\hline
\end{tabular}

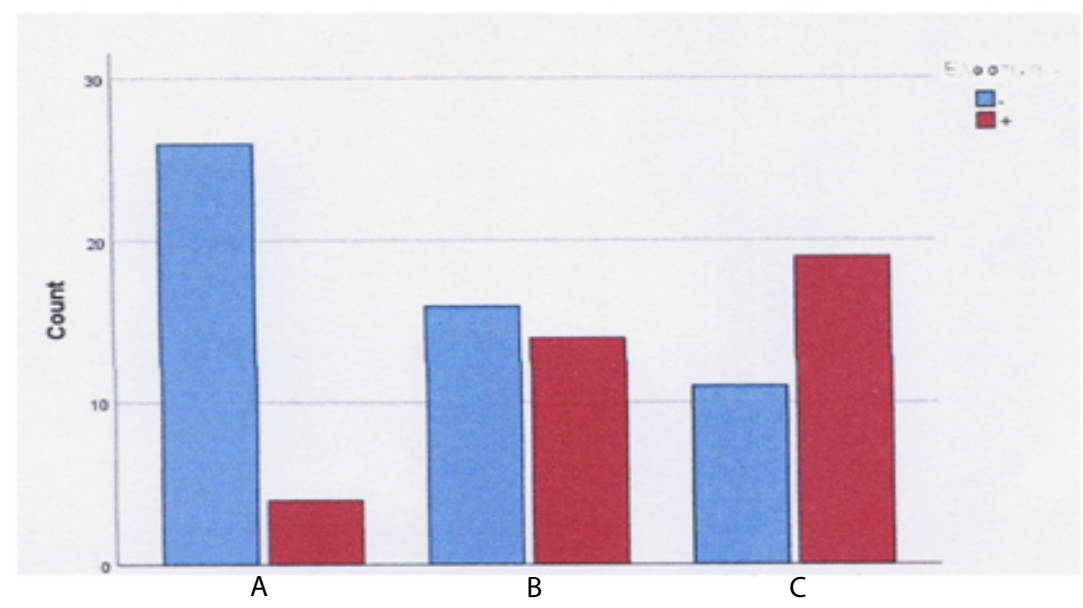

Graph 2. Elastin expression bar graph per Group (Group A: Patients with POP and SUI, Group B: Patients with POP and no SUI, Group C: controls)

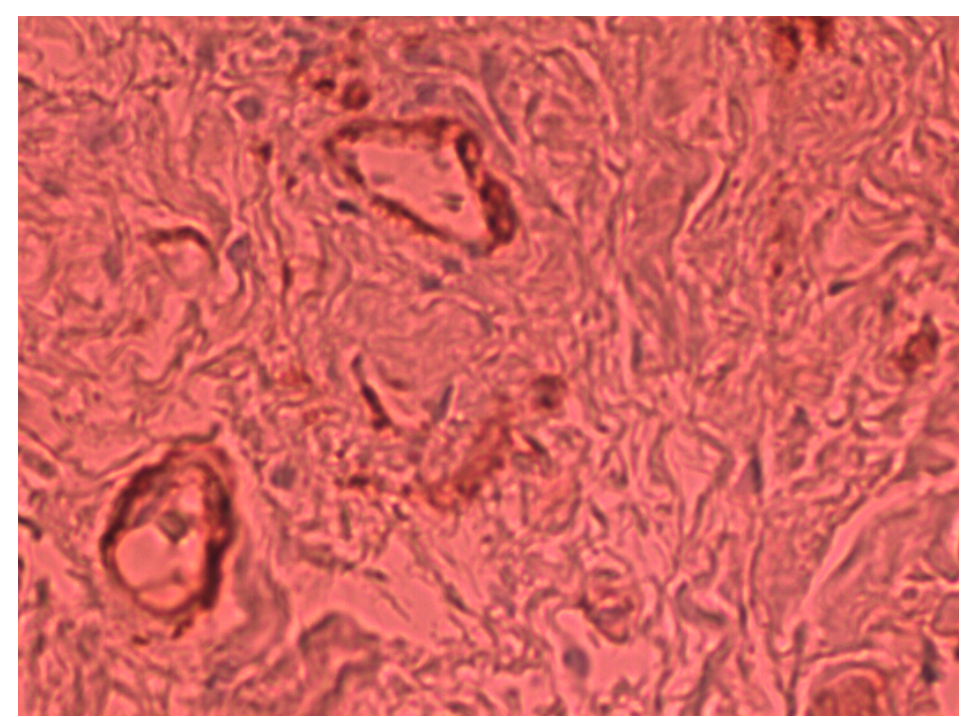

Figure 1. Extended and intensely positive immunoexpresion of tenascin with localization in stroma cells and close to vessels with mainly cellular and membranous localization from a 70-year-old patient with IV degree prolapse and stress urinary incontinence (Immunostaining of tenascin x200) 


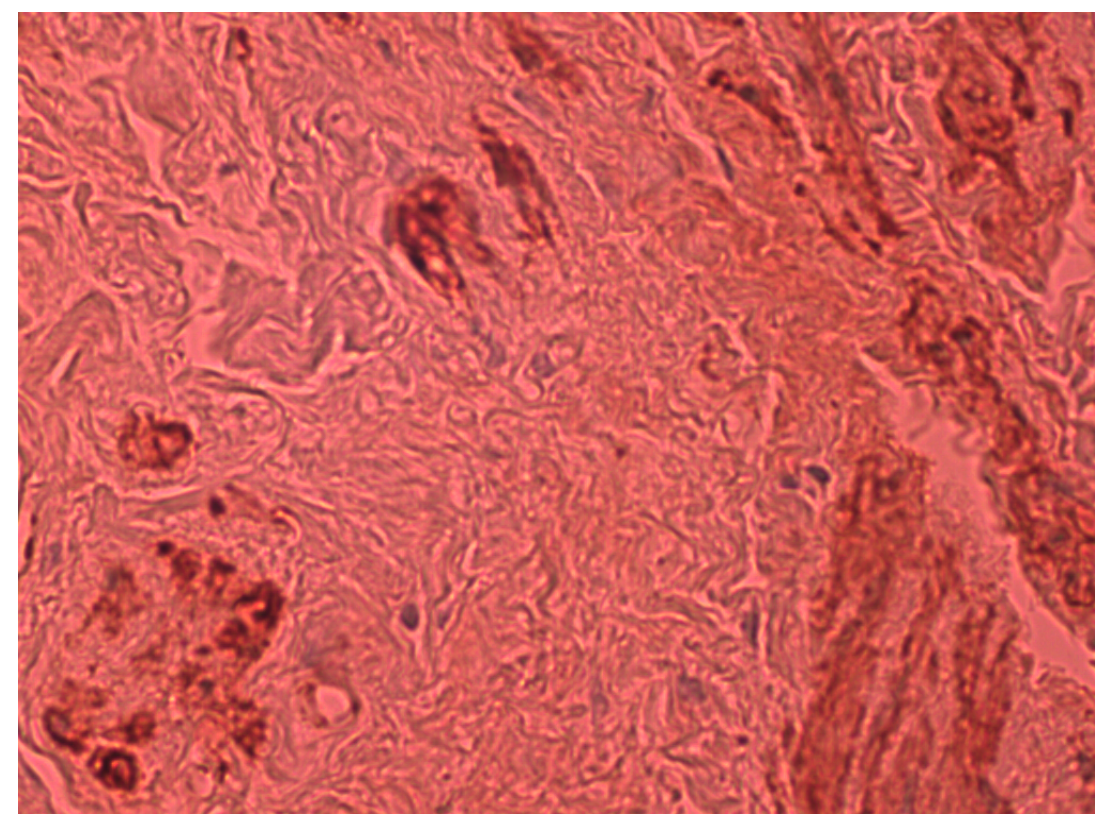

Figure 2. Negative-weakly positive tenascin immunoreaction in stromal cells. Local expression of tenascin in muscles / vessels is distinguished. The patient is 68 years old with no POP and no SUI (Immunostaining of tenascin x200)

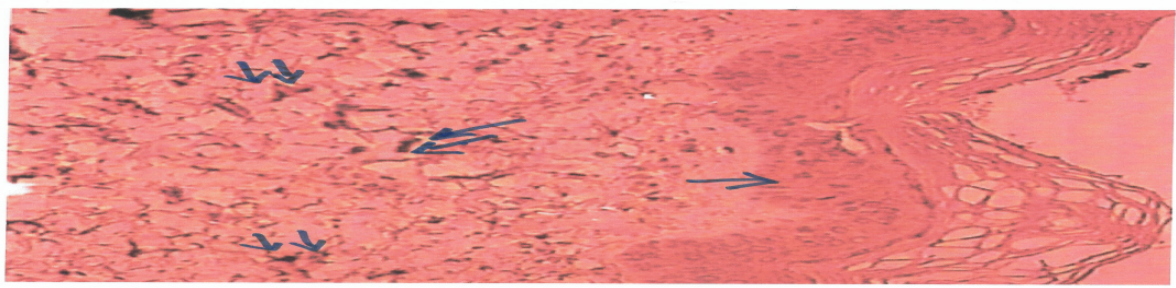

Figure 3. Histological section of vaginal wall, covered by squamous epithelium (single arrow), which shows a reticular elastin stromal immunoreaction (double arrows). (Immunoreaction x 200)

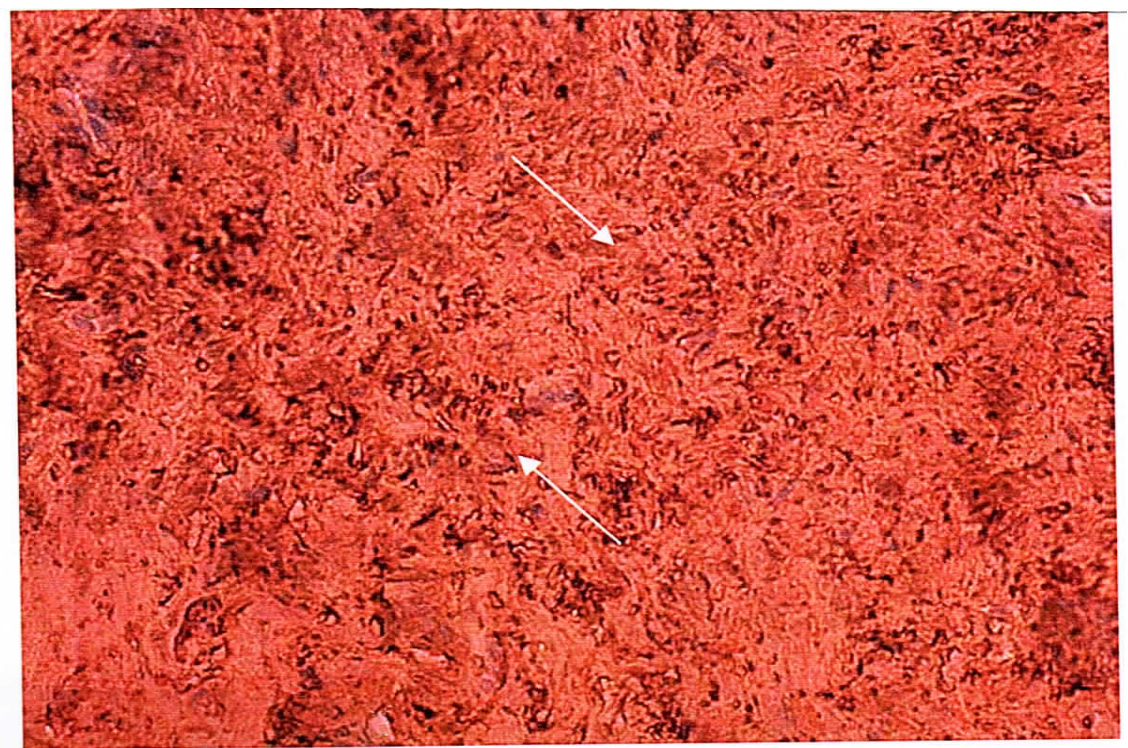

Figure 4. Histological section of vaginal wall showing strongly positive stromal elastin immunoreactions (arrows) in connection with muscle bundles (immunostaining x100) 
Tenascin-C expression is significantly increased in relaxed ligaments $[7,8]$. As an extracellular protein, tenascin-c is transiently present and is markedly upgraded in tissue rejuvenation [9]. Mechanical stretching has been reported to trigger the release of growth factors in an automatic or paracrine manner in a sufficient number of cells. These factors modify the rate of transcription of certain subsequent genes, including tenascin-C and other components of the extracellular matrix [10].

It has been shown that the expression levels of tenascin- $\mathrm{C}$ are affected by a variety of grown factors, including TGF- $\beta$, platelet growth factor (PGF) and cytokines as well. TGF- $\beta$ may stimulate the protein production of extracellular matrix (ECM) through fibroblasts. The results of the current study have shown that women with relaxation for which mechanical pressure and damage is required, had high tenascin expression levels in a significant percentage for Group A (76.7\%) and for group B (60\%), while the control group (Group C) had only $25.7 \%$. Although the exact support mechanism is not known, structure abnormalities of connective tissue or its repair mechanism may predispose women to prolapse [11].

Connective tissue and pelvic floor fascia may lose their strength as a result of age and loss of neuroendocrine signaling of the pelvic tissues [12]. An important part of the pelvic floor support system is the uteroscacral and cardinal ligaments and the fascia of pelvic floor [13].

Key elements of the tissue stability are the quantity, structural composition and organization of extracellular proteins, such as collagen, elastin and glycoproteins (tenascin, fibronectin, laminin etc.), as well as their receptors [14].

Relatively little data has been published regarding the role of glycoproteins in pelvic floor relaxation. Proteoglycans form a network around the collagen fibers that hold the fibers in place and resist compression by trapping water molecules. These include glycoproteins, such as tenascin, fibronectin, laminin etc., which provide a direct link to the surface of collagen or proteoglycans cells for the incorporation of the organized structure of the extracellular space. The relationship between the fibers and the substance that surrounds them is important for the natural properties of the specific tissue [15].

As previously mentioned, there are no available data in the literature regarding tenascin expression in connective tissue of pelvic floor. Tenascin is poorly distributed in the anterior cruciate ligaments, but its expression is clearly increased in ruptured tissues, indicating its involvement in the healing process of injured tissues [16].

The structure of glycoprotein tenascin was strongly labeled in all samples of our study analyzed. Weak expression was noted in tissues of women without prolapse (control group). The work of Goepel in 2008 came into the same results, stating that tenascin was substantially enhanced under the basal layer in all samples obtained from women with prolapse and especially in the connective tissue near the vessel walls [8].

Tenascin presence in pelvic floor relaxation is closely linked to alteration of extracellular proteins of supporting tissues [17]. Bone marrow mesenchymal cells have the potential to differentiate into a variety of cells, including osteoblasts, chondroblasts and adipose cells. Hence, the above cells have the potential to enhance the clinical effects of pelvic floor prolapse. Tenascin is a large glycoprotein which is present in the extracellular substance and is involved in morphogenetic movements of tissue formation and repair of the bone
[18]. Bhattacharrya et al. [19] have reported similar results, suggesting that tenascin leads to tissue fibrosis.

Conclusively, we result that the increased presence of tenascin levels in relaxed tissues and, particularly in the ligaments and fascia of the pelvic floor, is correlated to tissue injury. This trauma may have started with childbirth and the lack of estrogen following menopause can contribute to tissue breakdown.

The etiology of prolapse is affected by many environmental and genetic factors. Pregnancy and birth are considered key factors, as more than $60 \%$ of women with urinary incontinence correlate the symptom onset with the presence of prolapse, which could be attributed to the previously mentioned factors [20].

Elastin was expressed in the sample of this study in all tissues obtained from women without relaxation of pelvic floor. Elastic fibers were in close contact to smooth muscles fibers or were found in small quantities or were defragmented in women with pelvic organ prolapse. In tissues with positive elastin immune expression, the labeled structures were granular or fibrous and sometimes amorphous. These data suggest that elastin may play an important role in the pathophysiology of pelvic floor relaxation and that lack of proper expression may predispose women to development of relaxation. Significant differences of immune expression of elastin were observed. In postmenopausal women without relaxation, a normal pattern of elastin labeling was observed. In women with relaxation, however, elastin was either completely absent or show clear signs of fragmentation. Elastin plays an important role in the maintenance of ligaments, but the factors involved in the formation of elastic fibers are generally unknown [21]. Pelvic floor relaxation is associated with connective tissue abnormalities, while decrease of fibulin-5 expression and lysyl-oxidase- 1 at the uterosacral ligaments in postmenopausal women with pelvic organ prolapse shows that these substances are involved in the pathogenesis of pelvic tissue relaxation [20].

Yamamoto et al. showed that mRNA elastin levels were significantly reduced in fibroblast cultures of women with prolapse compared to the control group [22]. Until then, the role of elastin in uterine prolapse was not well defined. Lack of regeneration of functional elastic fibers in adults is a big problem and once this regenerative ability is lost, the restoration of normal function is not possible [23]. Adult tissues synthesize elastin as a response to recurred injury and many diseases, such as emphysema [21]. Adults, however, cannot rebuild the mechanisms for fitting fibers and, thus, restoring function. Elastic fibers seem to play an important role in providing elasticity and durability to some tissues, such as large arteries and, especially, to specific ligaments. The significant reduction of elastin labeling in patients with uterine prolapse noted in this study could lead to a lack of elastic fibers in the ligaments and, therefore, contribute to loss of suspension function of the uterine connective tissues [22]. Overall, the reduction of elastin immunization and increased tenascin synthesis may explain the loss of supporting function in the connective tissues of the uterus. These data indicate a possible role for these proteins in the pathogenesis and potential treatment of pelvic organ relaxation.

The mechanism by which pregnancy and vaginal delivery can lead to failure of pelvic organ support is not fully understood. Min et al. [24] investigating the expression of extracellular matrix in fibroblasts from the transverse cervical ligaments which, when exposed to various mechanical loads, showed that the alteration in the metabolism of the extracellular matrix contributed to the pathogenesis of pelvic organ prolapse. 


\section{References}

1. MacLennan AH (2000) The descent of woman. Climacteric 3: 225-226

2. Smith A, Bevan D, Douglas HR, James D (2013) Management of urinary incontinence in women: summary of updated NICE guidance. $B M J 347: 5170$. [Crossref]

3. Boreham MK, Wai CY, Miller RT, Schaffer JI, Word RN (2002) Morphometric analysis of smooth muscle in the anterior vaginal wall of women with pelvic organ prolapse. $\mathrm{Am}$ J Obstet Gynecol 187: 56-63.

4. Mescher AL (2013) Junqueira's basic histology: text and atlas. McGraw-Hill Medical 13th ed. New York.

5. Kerkhof MH, Hendriks L, Brölmann HAM (2009) Changes in connective tissue in patients with pelvic organ prolapse--a review of the current literature. Int Urogynecol $J$ Pelvic Floor Dysfunct 20: 461-474. [Crossref]

6. Yamamoto M, Aoyagi M, Akazawa K, Tajima S, Yamamoto K (1998) Decrease in p53 protein in cultured cardinal ligament fibroblasts from patients with prolapsus uteri. Cell Biol Int 22: 31-40. [Crossref]

7. Ewies AAA, Al-Azzawi F, Thompson J (2003) Changes in extracellular matrix proteins in the cardinal ligaments of post-menopausal women with or without prolapse: a computerized immunohistomorphometric analysis. Hum Reprod 18: 2189-2195. [Crossref]

8. Goepel C (2008) Differential elastin and tenascin immunolabeling in the uterosacral ligaments in postmenopausal women with and without pelvic organ prolapse. Acto Histochem 110: 204-209. [Crossref]

9. Lee UJ, Gustilo-Ashby AM, Daneshgari F, Kuang M, Vurbic D, et al. (2008) Lower urogenital tract anatomical and functional phenotype in lysyl oxidase like-1 knockout mice resembles female pelvic floor dysfunction in humans. Am J Physiol Physiol 295: F545-F555. [Crossref]

10. Chiquet M, Sarasa-Renedo A, Tunç-Civelek V (2004) Induction of tenascin-C by cyclic tensile strain versus growth factors: distinct contributions by Rho/ROCK and MAPK signaling pathways. Biochim Biophys Acta - Mol Cell Res 1693:193-204. [Crossref]

11. Norton P, Boyd C, Deak S (1992) Collagen synthesis in women with genital prolapse or stress urinary incontinence. Neurourol Urodyn 11: 300-301.
12. Smith AR, Hosker GL, Warrell DW (1989) The role of partial denervation of the pelvic floor in the aetiology of genitourinary prolapse and stress incontinence of urine. A neurophysiological study. Br J Obstet Gynaecol 96: 24-28. [Crossref]

13. DeLancey JOL (1992) Anatomic aspects of vaginal eversion after hysterectomy. Am J Obstet Gynecol 166: 1717-1728. [Crossref]

14. Ulmsten U, Falconer C (1999) Connective tissue in female urinary incontinence Curr Opin Obstet Gynecol 11: 509-515.

15. Buller JL, Thompson JR, W, et al (2001) Uterosacral ligament: description of anatomic relationships to optimize surgical safety. Obstet Gynecol 97: 873-879.

16. Neurath M (1993) Expression of tenascin, laminin and fibronectin following traumatic rupture of the anterior cruciate ligament. Z Orthop Ihre Grenzgeb 131: 168-172. [Crossref]

17. Zhao B, Hu M, Wu H, Ren C, Wang J, et al. (2017) Tenascin-C expression and it associated pathway in BMSCs following co-culture with mechanically stretched ligament fibroblasts. Mol Med Rep 15: 2465-2472.

18. Liu R, He Y, Li B, Liu J, Ren Y, et al. (2012) Tenascin-C produced by oxidized LDLstimulated macrophages increases foam cell formation through toll-like receptor-4. Mol Cells 34: 35-41. [Crossref]

19. Bhattacharyya S, Wang W, Morales-Nebreda L, Feng G, Wu M, et al. (2016) Tenascin-C drives persistence of organ fibrosis. Nat Commun 7: 11703. [Crossref]

20. Zhao B, Zhou J (2012) Decreased expression of elastin, fibulin-5 and lysyl oxidaselike 1 in the uterosacral ligaments of postmenopausal women with pelvic organ prolapse. J Obstet Gynaecol Res 38: 925-931. [Crossref]

21. Pierce RA, Mariani TJ, Senior RM (1995) Elastin in lung development and disease. Ciba Found Symp 192: 199-212.

22. Yamamoto K, Yamamoto M, Akazawa K, Tajima S, Wakimoto H, et al. (1997) Decrease in elastin gene expression and protein synthesis in fibroblasts derived from cardinal ligaments of patients with prolapsus uteri. Cell Biol Int 21: 605-611. [Crossref]

23. Comper WD (1996) Extracellular matrix. CRC Press.

24. Min J, Li B, Liu C, Guo W, Hong S, et al. (2017) Extracellular matrix metabolism disorder induced by mechanical strain on human parametrial ligament fibroblasts. Mol Med Rep 15: 3278-3284. [Crossref]

Copyright: (C2021 Liapis I. This is an open-access article distributed under the terms of the Creative Commons Attribution License, which permits unrestricted use, distribution, and reproduction in any medium, provided the original author and source are credited. 